\title{
Progress in 193nm Resists: Impact of The Development Process on Anhydride-Containing Resist Materials
}

\author{
Robert D. Allen, Carl E. Larson, Hoa D. Truong, Phillip J. Brock and Hiroshi Ito \\ IBM Almaden Research Center \\ 650 Harry Road \\ San Jose, CA 95120 \\ ndallen@almaden.ibm.com
}

\begin{abstract}
The impact of develop time and developer concentration was investigated for $193 \mathrm{~nm}$ resists based on "alternating" polymers of maleic anhydride and norbornene monomers (COMA), prepared by free radical copolymerization. The COMA materials show significant and unique performance dependence on the development process. The development process for COMA materials was found to be a powerful process variable. This paper suggests an explanation for these findings.
\end{abstract}

Keywords: cyclic olefins, maleic anhydride, COMA, resist dissolution, contrast curves

\section{Introduction}

As $193 \mathrm{~nm}$ resist technology approaches commercial use, performance questions remain. Outstanding materials issues including resist top and sidewall roughness, resist development contrast, etch resistance and overall imaging performance are the subjects of continuing concern. [1]

Polymer platform dependence on resist performance has been of keen interest as it applies to 193nm resist systems.[2] The three most widely studied polymer types are the alicyclic-modified acrylic resists, poly(cycloolefins), and the cyclic olefin-maleic anhydride (COMA) polymers.[3] The three backbone polymer structures are represented in Figure 1.

The acrylic (alicyclic-modified) resist systems have an advantage of being the most widely studied, as these systems were the first class of materials to be investigated in the early days of $193 \mathrm{~nm}$ lithography.[4-5] These materials are made via a straightforward polymerization process. The acrylic materials are quite transparent at $193 \mathrm{~nm}$ but are not robust to etch processes and may have metrology challenges, due to shrinkage in the SEM.[6] Additionally, the modem acrylic resist formulations apparently suffer from high side wall roughness problems.

The cyclic olefin (or poly(cycloolefin)) resists have received much attention recently, as these materials are extremely robust to etch processes.[7] They are capable of forming resists with high resolution imaging properties, but have been shown to have non-traditional development properties.[8]

The polymer family which is the subject of this paper, cyclic olefin-maleic anhydride (COMA) copolymers, show extremely interesting development properties, such as very low carboxylic acid contents required for positive-tone aqueous development without swelling, as was first disclosed in 1999.[2] Recent work uncovered the potential mechanism for this radical difference in development behavior: ring opening (hydrolysis) of the anhydride by aqueous base during development.[8] This effectively gives high ionization of the backbone, leading to high development rates, even at low starting carboxylic acid concentrations. The carboxylic acids in the polymer, resulting from ester deprotection by photoacid, or deliberately incorporated into the resist starting polymer [9], are thus "amplified" in this dissolution mechanism.

This paper describes and interprets the strong impact that the resist development process has on the cyclic olefin-maleic anhydride polymer family. The magnitude of this dependence will be characterized as a function of polymer polarity.

\section{Experimental}

Norbornene and substituted norbomene monomers readily polymerize by free radical polymerization (using AIBN, degassed polymerization solvents, etc.) when in the presence of maleic anhydride. The polymers formed are typically low-to-medium molecular weight $(\mathrm{Mw}=4000-8000)$, high $\mathrm{Tg}$ 
$\left(>200^{\circ} \mathrm{C}\right)$ polymers containing close to $50 \mathrm{~mole} \%$ maleic anhydride.[2]

Contrast curves were generated using the ISI microstepper (193nm, 0.6 NA) at the IBM Almaden Research Center. A method was developed to deliver in a controllable manner a very small incrementally higher dose, using an attenuated phase shifting mask (nonpattemed area) as a neutral density filter. This method provides dose increments of $0.13 \mathrm{~mJ} / \mathrm{cm}^{2}$ with high accuracy. This technique is quite useful to study contrast curves for very sensitive resists.

\section{Results and Discussion}

An interesting, possibly unique aspect of anhydride-based polymers is the impact of low levels (ca. $10-20 \%$ ) of carboxylic acid (either as-polymerized, or resulting from acid-catalyzed ester deprotection as is found in chemically amplified resists) on the polymer or resist dissolution. We have ahready reported on this unusual difference of anhydride polymers as compared to other polymer platforms used for $193 \mathrm{~nm}$ resists. [2] Figure 2 demonstrates two aspects of this low-acid dependency: rapid dissolution change over small change in carboxylic acid concentration, and high contrast resist behavior at low protecting group concentration.
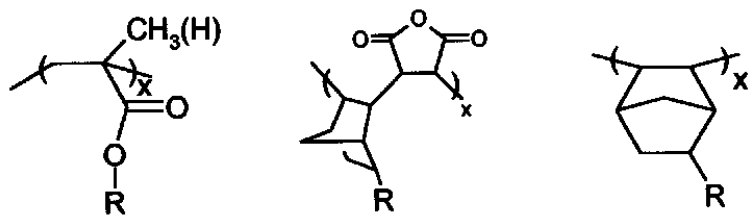

Figure 1. Chemical structure representation of the three main families of $193 \mathrm{~nm}$ resist polymers.

Based on these findings, the dissolution properties of COMA-based resists at low levels of exposure (i.e., "slightly deprotected") should be quite important. Furthermore, the response of the anhydride resist (and polymer backbone) to changing development conditions might be dependent on the type of COMA polymer used.

Two types of COMA resist compositions wre used in this study: "hydrophilic" resist formulations, which contain polar functional groups in ddition to the anhydride rings, and "hydrophobic" resist formulations, which do not contain polar functional groups other than the anhydride rings. The hydrophilic COMA resist, (e.g., containing low acid concentration nd/or polar functional groups attached to the polymer) (water contact angle = 60 degrees), has a finite unexposed thinming (dissolution) rate in $0.26 \mathrm{~N}$ TMAH. The hydrophobic COMA resist contain no acid or other polar groups in the resist (water contact angle $=\mathbf{8 0}$ degrees). These hydrophobic resist compositions show no thinning (unexposed) in developer. These two branches of the COMA resist family would be expected to show very different response to changing development conditions.
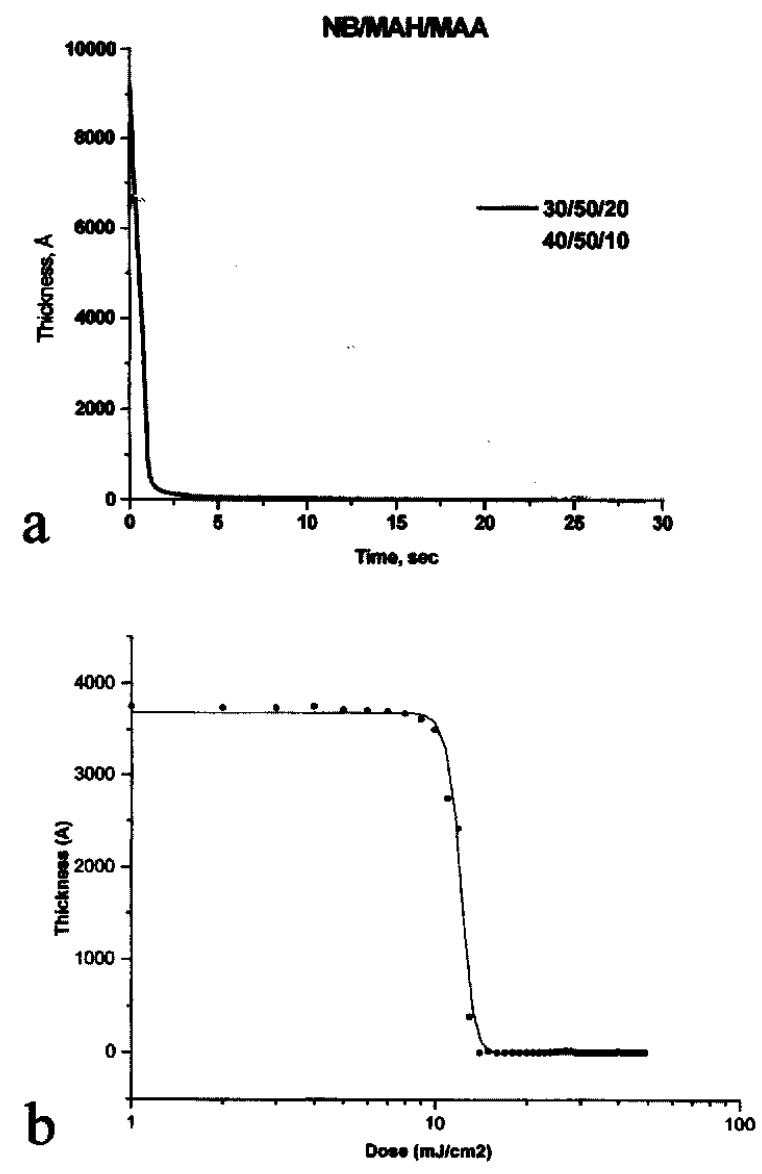

Figure 2. Dissolution response of alternating polymers. (a) Quartz Crystal Microbalance dissolution curves (left) of simple norbornene-maleic anhydride-carboxylic acid curves at 10 and $20 \%$ acid. (b) Resist contrast (right) curves of a COMA material containing ca. 15 mole\% t-butyl ester.

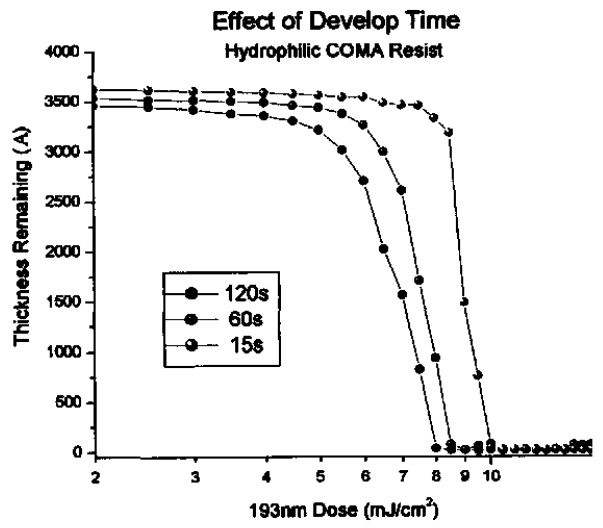

Figure 3. Impact of develop time for hydrophilic COMA resist on the resist response curves, using standard developer $(0.26 \mathrm{~N}$ TMAH). 
Figure 3 shows the impact of changing the develop time on the resist response curves ("contrast curves") for the hydrophilic COMA resist. Develop times ranging from as long as 120 seconds to as short as 15 seconds were used. First notice that thinning (unexposed dissolution) is a strong function of develop time (using standard developer). This is obvious, provided that the dissolution of these materials is linear with time. Figure 2(a) suggests that anhydride polymers show a linear development rate/time response, as found through quartz crystal microbalance dissolution studies. It is interesting that unexposed thinning can

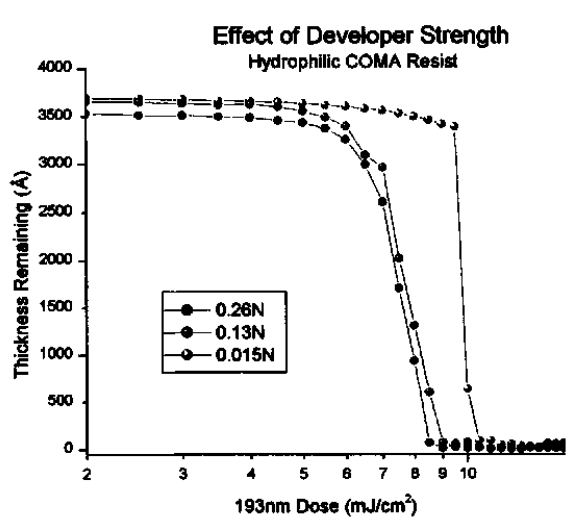

Figure 4. Developer strength impact on the resist response curves for a hydrophilic COMA resist

Tabb 1 : Summary of data from hydrophilic COMA resist response studies

\begin{tabular}{l}
\multicolumn{1}{c}{ Resist Process } \\
\begin{tabular}{|c|c|c|c|}
\hline Hydrophilic/0.26N & 120 & 8 & Gamma \\
\hline Hydrophilic/0.26N & 60 & 8.5 & 5.9 \\
\hline Hydrophilic/0.26N & 15 & 10 & 6.9 \\
\hline Hydrophilic/0.13N & 60 & 9 & 10.3 \\
\hline Hydrophilic/0.015N & 60 & 10.5 & 7 \\
\hline
\end{tabular}
\end{tabular}

Table 2: Summary of data from hydrophobic COMA resist response studies

\begin{tabular}{|c|c|c|c|}
\multicolumn{1}{c}{ Resist Process } & Dev. Time & 6 & Go $(\mathrm{mJ} / \mathrm{cm} 2)$ \\
\hline Hydrophobic/0.26N & 120 & 6.4 & $>20$ \\
\hline Hydrophobic/0.26N & 60 & 7.4 & $>20$ \\
\hline Hydrophobic/0.26N & 15 & 6.8 & $>20$ \\
\hline Hydrophobic/0.13N & 60 & 9 & $>20$ \\
\hline Hydrophobic/0.015N & 60 & & $>20$ \\
\hline
\end{tabular}

apparently be "turned off " through modulation for the development process. Also note that Eo (dose at initial complete development) is a strong function of develop time, as is contrast (see Table 1). This type of polymer (hydrophilic anhydride polymer), based on these simple observations, may be amenable to strong process control via the development process. These observations spawned another set of experiments: developer strength was changed over a large range $(0.015 \mathrm{~N}$ to $0.26 \mathrm{~N}$ TMAH). The resist response curves are shown in Figure 4.

Using lower strength developers for this hydrophilic COMA-class of resist materials allows for the control of resist response to an impressive degree. As shown in Figure 4, the resist dissolution at low doses is largely eliminated at concentrations of $0.13 \mathrm{~N}$ and below. Contrast is easily manipulated, with increasing contrast found at lower developer strengths (see Table 1). A medium contrast resist process can be elevated to a high contrast process by simply lowering the developer strength. The response of the resist at doses approching Eo is modified by tuming the developer strength. At "normal" $(0.26 \mathrm{~N})$ concentration, the resist dissolution gradually accelerates at doses approaching Eo (e.g., 5-7 $\mathrm{mJ} / \mathrm{cm}^{2}$ ). At low strength, the partially exposed resist development is highly surpressed $\left(5-9.5 \mathrm{~mJ} / \mathrm{cm}^{2}\right)$, causing almost "digital" contrast to appear.

The impact of development time on the hydrophobic COMA-class resulted in an unremarkable response, and is shown in Figure 5. All develop times lead to unmeasurably high contrast (above the dose increment used in the experiment $\left(0.2 \mathrm{~mJ} / \mathrm{cm}^{2}\right)$, presumably due to the higher acid concentrations required for positive-tone development. A significant shift in Eo was observed (see Table 2). A remarkable fact is that so little influence was seen, especially in relation to the hydrophilic materials discussed previously. This is presumably due to the negligible impact of low level exposure (said another way, low levels of carboxylic acid) on hydrophobic, anhydride- 
containing resist materials. More carboxylic acid is apparently required to cause solubility change in this branch of the COMA family.

The use of lower strength developers in this class of materials (hydrophobic COMA) showed a subtantial change in Eo, but again, only a subtle change in resist response curves (see Table 2 and Figure 6, respectively). All conditions used lead to high contrast. It may be remarkable that in anhydride polymers, even very weak developer leads to complete dissolution for hydrophobic resists. Although contrasts are universally high, low developer strength leads to non-digital (i.e., measurable) values.

The assembled data suggests that an unusual feature of these COMA resists is that a superposition in develop time and developer concentration may exist. It is especially easy to see this effect in the hydrophilic COMA resist response. The response curves at short development times and weak developer appear to be very similar. That is what may be different about certain 193mm resist backbone chemistries: carboxylic acid polymers can dissolve in aqueous base rapidly, regardless of developer concentration. Resists based on COMA polymers show amplified dissolution effects as compared to other materials. The main factor that changes when the development process is modified (or the polarity of the polymer) is the critical concentration of carboxylic acid required for development. It appears that the lithography process can utilize this effect to influence and possibly control contrast.

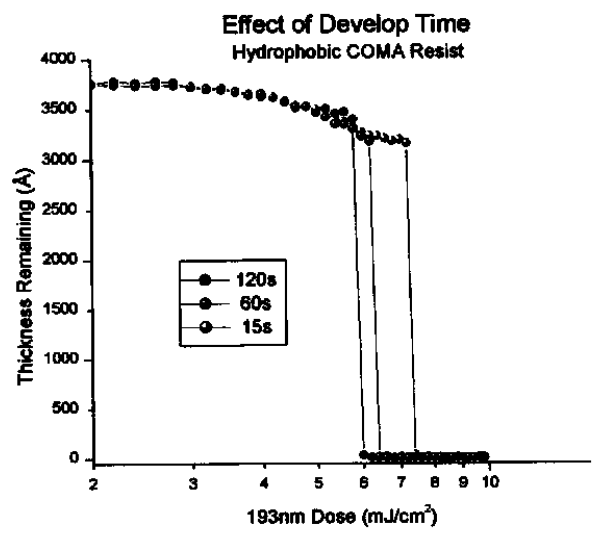

Figure 5. Impact of develop time on the resist response curves for a hydrophobic COMA resist, using standard $(0.26 \mathrm{~N}$ TMAH) developer.

The role of the development process on the imaging performance of selected COMA $193 \mathrm{~nm}$ resists is the topic of current interest, given the interesting results presented here. A comparison of COMA with other platforms is another topic of current interest.

\section{Conclusion}

This paper invetigated the impact of the devel-opment process variables (develop time using standard developer and develop strength at a constant time) for cyclic olefin/maleic anhy-dride (COMA) based resists. Two separate responses were found. Strong response was found for anhydride polymers containing additional polar functionalities (hydrophilic class), and weaker response was found with materials containing no additional hydrophilic functionalities, beyond the anhydride ring itself (hydrophobic class).

Both contrast and Eo can be manipulated to a surprising extent with the hydophilic class of COMA materials. This control is thought to be modulated by the response of the partially exposed resist material (at doses below Eo). Weaker developer concentration or shorter development time minimizes the solubility of this material, thus boosting contrast. Only Eo could be manipulated in the hydropho-bic class of COMA materials (i.e., carboxylic acid concentration required for resist dissolu-tion), while contrast is not affected as gamma is quite high $(>20)$ for these materials. The correlation of these basic contrast and dis-solution properties with imaging in these classes of COMA materials is the subject of our ongoing investigation.

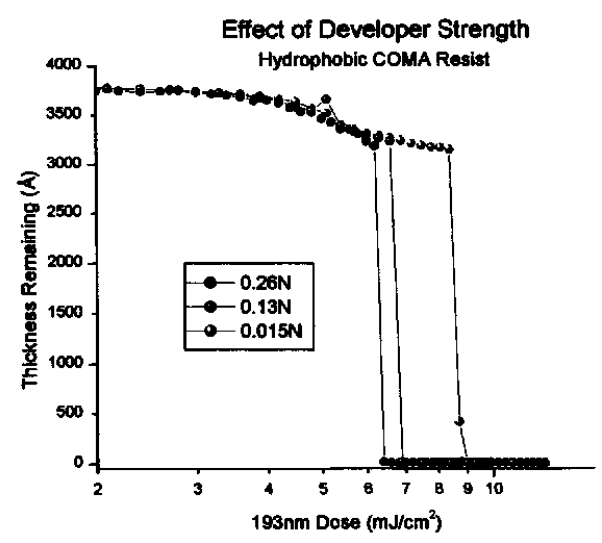

Figure 6. Impact of developer strength on the resist response curves for a hydrophobic COMA resist.

\section{References}

1. G. Amblard, J. Byers, W-D. Domke, G. Rich, V. Graffenberg, S. Patel, D. Miller and G. Perez, Proc. SPIE, 3999 (2000), in press.

2. R Allen, T. Wallow, J. Opitz, C. Larson, R. DiPietro, R. Sooriyakumaran, P. Brock, G. Breyta, D. Hofer, S. Jayaraman, R. Vicari, K. Hullihen, L. Rhodes, B. Goodall and R. Schick, Jo. Photopolym. Sci. Technol., 11(3), 475 (1998); R. Allen, J. Opitz, H. Ito, T. Wal-low, D. Casmier, C. Larson, R. Sooriyakuma-ran, D. Hofer 
and P. Varanasi, Jo. Photopolym. Sci. Technol., 12(3), 501 (1999).

3. R. D. Allen, W. E. Conley, and R. R. Kunz, "DeepUV Resist Technology," Chp. 4 in Handbook of Microlithography, Ed. P. Rai-Choudhury, SPIE Press, 1997.

4. K. Nozaki, K. Watanabe, E. Yano, A. Kota-chi, S. Takechi, and I. Hanyu, Jo. Photopolym. Sci. Technol., 9(3), 509 (1996).

5. R. Allen, I. Wan, G. Wallraff, R. DiPietro, D. Hofer and R. Kunz, Jo. Photopolym. Sci. Technol, 8(4), 623 (1995).
6. R. Allen, J. Opitz, C. Larson, T. Wallow and D. Hofer, "The Potential of $193 \mathrm{~nm}$ Photore-sists" Microlithography World, 5 (Winter 1999).

7. U. Okoroanyanwu, T. Shimokawa, J. Byers, and C.G. Willson, Chem. Mater., 10(11), 3319 (1998); P. Varanasi, et al., Proc. SPIE, 3678, 51 (1999).

8. H. Ito, et al., Proc. SPIE, 3999 (2000) in press.

9. T. Wallow, F. Houlihan, O. Nalamasu, E. Chandross, T. Neenan and E. Reichmanis, Proc. SPIE, 2724, 355 (1996); F. Houlihan, T. Wallow, O. Nalamasu, and E. Reichmanis, Macromolecules, 30(21), 6517 (1997). 